\title{
Lymphome de Burkitt : présentation d'une localisation maxillaire
}

\author{
Lamia Kissi, Rajaa El Bouihi, Mohamed Bouzoubaa, Mouna Lamchahab, \\ Ihsane Ben Yahya, Ahmed Alaoui
}

Faculté de Médecine dentaire, rue Abou Alaa Zahr, 21100 Casablanca, Maroc

lamiakissi@yahoo.fr

Le lymphome de Burkitt est une forme de lymphome malin non-hodgkinien dû à l'évolution et à la prolifération malignes de cellules lymphoïdes de type B. Classiquement, on définit trois variantes cliniques: la forme endémique ou africaine, liée au virus Epstein Barr (EBV), qui touche les maxillaires, la forme associée à l'immunodéficience due au VIH et la forme sporadique qui intéresse surtout l'abdomen. Il peut également se développer dans la sphère ORL et plus rarement dans d'autres localisations (orbite, rein, os...) (Patte 2010). Il représente environ 7-8 \% des tumeurs malignes chez les sujets de 15 à 19 ans (Patte et al. 2007). Les sujets de sexe masculin sont plus touchés (Freitas et al. 2008).

Le cas rapporté concerne un jeune homme d'une vingtaine d'années, adressé pour des tuméfactions gingivales évoluant depuis 1 mois, qui seraient apparues après des extractions dentaires. Ce patient, sans antécédents particuliers, est fébrile et présente un amaigrissement, des sueurs nocturnes et des douleurs osseuses dans les membres inférieurs. La palpation des aires ganglionnaires révèle la présence d'adénopathies cervicales.

L'examen clinique endobuccal montre, dans la région molaire, l'existence de tuméfactions gingivales, maxillaires et mandibulaires, molles et légèrement douloureuses à la palpation, associées à des mobilités dentaires. La radiographie panoramique dentaire révèle des lyses osseuses dans les régions molaires gauches avec opacité du sinus maxillaire homolatéral; la TDM un processus tumoral comblant le sinus maxillaire gauche en totalité.

Les examens biologiques sont normaux en dehors d'une VS très élevée. L'examen anatomopathologique, complété par un examen en immunohistochimie, montre qu'il s'agit d'un lymphome de Burkitt. Le bilan d'extension conclut à un lymphome de Burkitt stade IV (groupe C) selon la classification d'Ann Arbor.

La prise en charge a consisté en une chimiothérapie selon les protocoles suivants : une $1^{\text {ère }}$ cure de COP (cyclophosphamide, vincristine, prednisone) suivie de deux cures de COPADEM (cyclophosphamide, vincristine, doxorubicine, prednisone). Un mois après le début du traitement, on observe une rémission complète des lésions buccales.

Le lymphome de Burkitt est une tumeur rare et agressive. Il représente un véritable problème de santé publique où le médecin dentiste occupe une place importante dans le dépistage précoce qui permet une prise en charge rapide et appropriée, seul garant de la guérison (Koffi 2010). Les mécanismes responsables de la transformation maligne restent mal connus. Le diagnostic positif repose sur la biopsie de la masse tumorale ou la ponction de la moelle osseuse révélant la présence de cellules tumorales. Le traitement repose sur une chimiothérapie intensive. Dans le diagnostic différentiel, on doit évoquer un abcès dentaire, une ostéomyélite, un fibrome ossifiant, un ostéosarcome, un granulome éosinophile, un neuroblastome ou une dysplasie fibreuse (Ugar et al. 2006). 